\title{
Nivolumab-induced cholangitis in patients with non-small cell lung cancer: Case series and a review of literature
}

\author{
HIROKI IZUMI, MASAHIRO KODANI, JUN KURAI, KENICHI TAKEDA, RYOTA OKAZAKI, \\ KOHEI YAMANE, YASUHIKO TERUYA, AKIHIRO YAMAMOTO, YURIKO SUEDA, \\ MASAAKI YANAI, NATSUMI TANAKA, TOMOHIRO SAKAMOTO, KOSUKE YAMAGUCHI, \\ HARUHIKO MAKINO, TADASHI IGISHI and AKIRA YAMASAKI
}

Division of Medical Oncology and Molecular Respirology, Faculty of Medicine, Tottori University, Yonago, Tottori 683-8504, Japan

Received March 14, 2019; Accepted June 21, 2019

DOI: $10.3892 / \mathrm{mco} .2019 .1923$

\begin{abstract}
Immune checkpoint inhibitors (ICIs), including nivolumab, have exhibited substantial benefits in the treatment of several types of cancers. However, treatment with ICIs is often accompanied by immune-related adverse events (irAEs), and a clear understanding of the precise indications and management of irAEs is important for harnessing the full potential of these agents. While skin- or gastrointestinal-associated irAEs have been relatively well studied, there are few reports regarding nivolumab-induced cholangitis. We retrospectively reviewed data from patients with advanced or recurrent non-small cell lung cancer who were treated with nivolumab between December 2015 and December 2018 at Tottori University in Japan. Among the 59 patients, we identified four patients who experienced nivolumab-induced cholangitis. Of these four patients, stable disease (SD) was observed in two patients $(50 \%)$, while partial response (PR) was achieved in two patients $(50 \%)$ under nivolumab treatment. Patients were treated with corticosteroid alone $(n=2)$ or in combination with mycophenolate mofetil (MMF) $(\mathrm{n}=2)$; these treatments resulted in improvements in nivolumab-induced cholangitis in three patients. In conclusion, the present retrospective study identified four cases of nivolumab-induced cholangitis.
\end{abstract}

Correspondence to: Dr Hiroki Izumi, Division of Medical Oncology and Molecular Respirology, Faculty of Medicine, Tottori University, 36-1 Nishi-cho, Yonago, Tottori 683-8504, Japan

E-mail: hiroizu0211@gmail.com

Abbreviations: PD-1, programmed death-1; PD-L1, PD-1 ligand 1; NSCLC, non-small cell lung cancer; irAE, immune-related adverse event; HFT, hepatobiliary function test; PR, partial response; $\mathrm{SD}$, stable disease; $\mathrm{PD}$, progressive disease; RR, response rate; $\mathrm{DCR}$, disease control rate; MMF, mycophenolate mofetil; mPSL, methylprednisolone

Key words: non-small cell lung cancer, nivolumab, immune-related adverse event, cholangitis, mycophenolate mofetil
The combination of corticosteroid and MMF was effective in two cases with grade 4 nivolumab-induced cholangitis. Further reports are needed to establish the optimal management of patients with this irAE.

\section{Introduction}

Lung cancer is the most frequent cancer, with $>220,000$ estimated new cases per year and accounting for $>140,000$ estimated cancer deaths in the US (1). Despite the advance of therapeutic strategy for this malignancy, the prognosis of patients with advanced- or recurrent lung cancer is still poor, with 5-year survival estimates of $\sim 5 \%$ (1).

The programmed death-1 (PD-1) receptor, which is expressed on activated $\mathrm{T}$ cells, is engaged by the ligands PD-1 ligand 1 (PD-L1) and PD-L2, which are expressed by tumor cells and infiltrating immune cells (2). The interaction of PD-1 with PD-L1 and PD-L2 inhibits T-cell activation and promotes tumor immune escape $(3,4)$. Nivolumab is a fully human IgG4 anti-PD-1 antibody that disrupts PD-1-mediated signaling and restores antitumor immunity (5). Immune checkpoint inhibitors (ICIs) including nivolumab have been approved for the treatment of several types of cancer, including advanced non-small cell lung cancer (NSCLC), on the basis of recent clinical trials demonstrating that it prolongs survival compared with that of cytotoxic chemotherapy $(6,7)$. However, treatment with ICIs such as nivolumab is often accompanied by immune-related adverse events (irAEs). A clear understanding of the precise indications and management of irAEs is important for harnessing the full potential of these agents. The recommended management of irAEs is based on clinical experience or in accordance with procedures for the management of autoimmune disease. While skin- or gastrointestinal-related irAEs have been relatively well studied (8), there are few reports regarding nivolumab-induced cholangitis. Thus, few information is available with regard to clinical manifestation and treatment outcome of this rare type of irAE. Herein, we report a series of four cases of nivolumab-induced cholangitis with NSCLC and review the literature on cholangitis induced by nivolumab. 


\section{Patients and methods}

We retrospectively reviewed the data from patients with advanced or recurrent NSCLC who were treated with nivolumab between December 2015 and December 2018 at Tottori University Hospital (Yonago, Japan). Nivolumab $(3.0 \mathrm{mg} / \mathrm{kg})$ monotherapy was administered intravenously every 2 weeks until disease progression or irAE occurrence. Treatment-related select adverse events those were potentially immune related (skin, gastrointestinal, endocrine, hepatic, hypersensitivity/infusion reaction, pulmonary, and renal) without any other causes were defined as irAEs $(6,7)$. PD-L1 expression was assessed using the commercially available PD-L1 IHC 22C3 pharmDx assay (Dako North America). Expression was categorized according to the tumor proportion score (TPS). A PD-L1 TPS of $<1 \%$ was considered negative.

Clinicopathological data, including the duration of nivolumab treatment, response to nivolumab treatment, laboratory abnormalities, radiological findings, and management of nivolumab-related cholangitis, were analyzed. The data regarding tumor specimens (histology, PD-L1 expression, and driver gene alteration) was obtained at the diagnosis of disease. The other data was obtained when nivolumab treatment was administered. Computed tomography (CT) of the chest, abdomen, and/or pelvis was performed prior to and every 2 months during nivolumab treatment. Patients underwent abdominal CT and additional imaging tests, including endoscopic retrograde cholangiopancreatography (ERCP), endoscopic ultrasonography (EUS), or magnetic resonance cholangiopancreatography (MRCP) upon abnormal hepatobiliary function test (HFT) results during or after nivolumab treatment. Cholangitis was defined as HFT abnormalities with a dominant increase in biliary enzymes (alkaline phosphatase and $\gamma$-glutamyl transferase) compared to transaminase (aspartate aminotransferase and alanine aminotransferase) levels. Adverse events were graded according to the National Cancer Institute Common Terminology Criteria for Adverse Events (CTCAE), version 4.0. In this study, we particularly focused on describing the clinical manifestation and treatment outcome of nivolumab-induced cholangitis, one of rare irAEs. In that sense, we performed computer searches of the literature using the PubMed database, with the following keywords: 'non-small cell lung cancer'; 'nivolumab'; 'cholangitis'; 'biliary injury'. Meeting abstracts were excluded from analysis, as were articles written in languages other than English.

The patient characteristics and response to nivolumab treatment were compared between patients with and without irAEs using Mann-Whitney test for age, and Chi-square test for the other characteristics, respectively. Statistical analyses were performed using PASW Statistics 19 (IBM SPSS Statistics). $\mathrm{P}<0.05$ was considered to indicate a statistically significant difference.

\section{Results}

Clinicopathological characteristics of patients with nivolumab-induced cholangitis. A total of 59 patients with advanced or recurrent NSCLC were treated with nivolumab between December 2015 and December 2018 at Tottori University Hospital. Among these 59 patients, we identified four patients who experienced nivolumab-related cholangitis, yielding an incidence rate of $6.8 \%$. irAEs other than cholangitis that resulted in discontinuation of nivolumab were observed in five $(9.8 \%$ ) patients (rash, $\mathrm{n}=2$; interstitial lung disease, $\mathrm{n}=1$; hypopituitarism, $n=1$; diarrhea, $n=1$ ). No patients experienced irAEs in multiple organs.

The baseline characteristics of patients are summarized in Table I. There was no significant difference between patients with and without irAEs. The ages of the four patients with cholangitis ranged from 55 to 82 years (median age, 78 years). Among the four patients, three had adenocarcinoma, and the other patient had squamous cell carcinoma. Metastases to the liver upon the administration of nivolumab were observed in one patient (case 4). None of the four patients had any history of cholangitis or pancreatitis prior to the administration of nivolumab treatment. PD-L1 expression was assessed in $31(52.5 \%)$ out of the 59 patients. Among the patients tested, all four patients with any irAE were positive for PD-L1 expression (1-49\%, $\mathrm{n}=1 ; \geq 50 \%, \mathrm{n}=3)$. Among the 27 patients without irAEs in whom PD-L1 expression was assessed, 14 (51.9\%) were positive for PD-L1 expression $(1-49 \%, \mathrm{n}=8 ; \geq 50 \%, \mathrm{n}=6)$. Driver gene alterations were detected in three patients [KRAS mutation: Cases 2 and 3; MET exon 14 skipping: Case 4; gene alterations were not investigated in the patient with squamous cell carcinoma (case 1)].

Nivolumab was administered as second-line therapy in two patients (cases 2 and 3) and third-line therapy in the other two patients (cases 1 and 4). The best response to nivolumab treatment was partial response (PR), which was observed in two patients (cases 1 and 3), while stable disease (SD) was observed in the other two patients. Thus, the response rate (RR) and disease control rate (DCR) in patients with cholangitis were 50 and $100 \%$, respectively. Among five patients with irAEs other than cholangitis, PR was observed in four $(80.0 \%)$ patients and PD in one (20.0\%) patient. The RR and DCR in the 50 patients without any irAEs were 27.5 and $60.8 \%$, respectively. The RR in patients with irAEs was significantly higher than that in patients without irAEs (66.7 vs. $27.5 \%, \mathrm{P}=0.02)$.

Clinical manifestation of nivolumab-related cholangitis. HFT abnormalities occurred after 2-24 cycles (median, 6.5 cycles) of nivolumab treatment. Symptoms were fairly mild and non-specific, including epigastric pain and appetite loss at the time HFT abnormalities occurred. Biliary enzymes were dominantly increased in all cases, with peak alkaline phosphatase levels of 3,029-9,118 U/1 (reference range, $<322 \mathrm{U} / \mathrm{l})$ and peak $\gamma$-glutamyl transpeptidase levels of 829-3,442 U/1 (reference range, <32 U/l), compared with levels of hepatic enzymes, with peak aspartate aminotransferase levels of 284-682 U/1 (reference range, $<30 \mathrm{U} / \mathrm{l}$ ) and peak alanine aminotransferase levels of 235-953 U/1 (reference range, $<23 \mathrm{U} / \mathrm{l})$. A moderate increase in total bilirubin levels of $\leq 3.8 \mathrm{mg} / \mathrm{dl}$ was also observed.

There were no recent changes in medications, aside from nivolumab. Serological tests for viral hepatitis, autoimmune hepatitis, or cholangitis were performed to exclude other causes of cholangitis in all four patients. None of the four patients were positive for hepatitis B virus (HBV), hepatitis C virus (HCV), $\mathrm{IgG} 4$, anti-smooth muscle antibody, or anti-mitochondrial 
Table I. Characteristics of patients with and without irAEs (nivolumab-induced cholangitis or others).

\begin{tabular}{|c|c|c|c|c|}
\hline Characteristic & $\begin{array}{l}\text { Patients with } \\
\text { cholangitis } \\
(n=4), n(\%)\end{array}$ & $\begin{array}{l}\text { Patients with irAEs } \\
\text { other than cholangitis } \\
\quad(n=5), n(\%)\end{array}$ & $\begin{array}{l}\text { Patients without } \\
\text { any irAEs } \\
(\mathrm{n}=50), \mathrm{n}(\%)\end{array}$ & P-value \\
\hline Age, range (median) & $55-82(78)$ & $62-79(71)$ & 43-91 (66) & 0.16 \\
\hline Sex & & & & 0.98 \\
\hline Male & $2(50)$ & $5(100)$ & $39(78.0)$ & \\
\hline Female & $2(50)$ & 0 & $11(22.0)$ & \\
\hline Histology & & & & 0.97 \\
\hline Adenocarcinoma & $3(75)$ & $2(40.0)$ & $29(58.0)$ & \\
\hline Squamous cell carcinoma & $1(25)$ & $2(40.0)$ & $17(34.0)$ & \\
\hline LCNEC & 0 & 0 & $1(2.0)$ & \\
\hline NSCLC-NOS & 0 & $1(20.0)$ & $3(6.0)$ & \\
\hline Performance status & & & & 0.37 \\
\hline $0-1$ & $3(75.0)$ & $3(60.0)$ & & \\
\hline $2-4$ & $1(25.0)$ & $2(40.0)$ & & \\
\hline Liver metastasis & & & & 0.11 \\
\hline Yes & $1(25)$ & $1(20.0)$ & $3(6.0)$ & \\
\hline No & $3(75)$ & $4(80.0)$ & $47(94.0)$ & \\
\hline Brain metastasis & & & & 0.84 \\
\hline Yes & 0 & $3(60.0)$ & $15(30.0)$ & \\
\hline No & $4(100)$ & $2(40.0)$ & $35(70.0)$ & \\
\hline PD-L1 expression (tumor proportion score), \% & & & & 0.07 \\
\hline$<1$ & 0 & 0 & $13(26.0)$ & \\
\hline $1-49$ & 0 & $1(20.0)$ & $8(16.0)$ & \\
\hline$>50$ & $1(25.0)$ & $2(40.0)$ & $6(12.0)$ & \\
\hline Unknown & $3(75.0)$ & $2(40.0)$ & $23(46.0)$ & \\
\hline Driver gene alteration & & & & 0.09 \\
\hline$E G F R$ & 0 & 0 & $5(10.0)$ & \\
\hline$A L K$ & 0 & 0 & 0 & \\
\hline$K R A S$ & $2(50)$ & $1(20.0)$ & $4(8.0)$ & \\
\hline Others & $1(25)$ & 0 & $1(2.0)$ & \\
\hline Not detected & 0 & $2(40.0)$ & $24(48.0)$ & \\
\hline Unknown & $1(25)$ & $2(40.0)$ & $16(32.0)$ & \\
\hline Timing of nivolumab & & & & 0.94 \\
\hline 2 nd & $2(50)$ & $1(20.0)$ & $16(31.4)$ & \\
\hline 3rd or later & $2(50)$ & $4(80.0)$ & $34(68.6)$ & \\
\hline Response to nivolumab & & & & 0.02 \\
\hline $\mathrm{CR}+\mathrm{PR}$ & $2(50)$ & $4(80.0)$ & $14(27.5)$ & \\
\hline SD & $2(50)$ & 0 & $17(33.3)$ & \\
\hline PD & 0 & $1(20.0)$ & $18(35.3)$ & \\
\hline $\mathrm{NE}$ & 0 & 0 & $1(2.0)$ & \\
\hline Smoking & & & & 0.94 \\
\hline Current or former & $3(75.0)$ & $5(100)$ & $44(88.2)$ & \\
\hline Never & $1(25)$ & 0 & $6(11.8)$ & \\
\hline
\end{tabular}

LCNEC, large cell neuroendocrine carcinoma; irAE, immune-related adverse event; NSCLC-NOS, non-small cell lung cancer-not otherwise specified; PD-L1, programmed death-ligand 1; CR, complete response; PR, partial response; SD, stable disease; PD, progressive disease; NE, not evaluable.

antibody. Three (75\%) patients were positive for anti-nuclear antibody (ANA) at the time of diagnosis of nivolumab-induced cholangitis. There were no data available regarding auto-antibody status before treatment with nivolumab. 
Table II. Clinicopathological characteristics of NSCLC patients with ICI-induced cholangitis.

\begin{tabular}{ccccccccc}
\hline Case & Author, year & $\begin{array}{c}\text { Age, } \\
\text { years }\end{array}$ & Sex & Histology & $\begin{array}{c}\text { Timing of } \\
\text { nivolumab }\end{array}$ & $\begin{array}{c}\text { No. of } \\
\text { cycles }\end{array}$ & $\begin{array}{c}\text { Best response to } \\
\text { treatment with ICI }\end{array}$ & (Refs.) \\
\hline 1 & - & 81 & F & Sq & 3rd line & 24 & PR & Present study \\
2 & - & 75 & M & Ad & 2nd line & 2 & SD & Present study \\
3 & - & 55 & M & Ad & 2nd line & 11 & PR & Present study \\
4 & - & 82 & F & Ad & 3rd line & 2 & SD & Present study \\
5 & Kawakami et al, 2017 & 64 & M & Ad & 2nd line & 9 & PR & $(10)$ \\
6 & Kawakami et al, 2017 & 73 & F & Sq & 4th line & 6 & PR & $(10)$ \\
7 & Kawakami et al, 2017 & 82 & F & Sq & 2nd line & 12 & SD & $(10)$ \\
8 & Gelsomino et al, 2017 & 79 & M & NSCLC-NOS & NA & 4 & PD & $(11)$ \\
9 & Kashima et al, 2018 & 63 & M & Ad & 2nd line & 4 & N/A & $(12)$ \\
10 & Kuraoka et al, 2018 & 69 & M & Ad & 3rd line & 3 & N/A & $(13)$ \\
\hline
\end{tabular}

ICI, immune checkpoint inhibitors; F, female; M, male; Sq, squamous cell carcinoma; Ad, adenocarcinoma; NSCLC-NOS, non-small cell lung cancer-not otherwise specified; PR, partial response; $\mathrm{SD}$, stable disease; N/A, not available.

Imaging results. Additional abdominal CT, ERCP, and EUS scans were performed to diagnose the cause of the hepatobiliary abnormalities. Characteristic imaging results are provided in Table II. In three cases (cases 1, 3, and 4), we identified common imaging patterns, including periportal edema (PE), extrahepatic biliary duct (EHD) dilatation, and hypertrophy of EHD, without any obstructive regions, including stones or malignancy (Fig. 1).

Clinical course after onset of nivolumab-induced cholangitis. From these clinical manifestations, serological tests, and imaging results, we diagnosed these four cases with nivolumab-induced cholangitis. Although nivolumab treatment was immediately postponed, HFT abnormalities did not improve, but rather worsened. Immunosuppressive treatment with systemic corticosteroid [methylprednisolone (mPSL), $2.0 \mathrm{mg} / \mathrm{kg}$ daily] was administered to all patients, and cases 1 and 3 were additionally treated with mycophenolate mofetil (MMF) based on the recommendation of the European Society for Medical Oncology (ESMO) clinical practice guideline (9). The time course of immunosuppressive treatment and HFT in all four patients is summarized in Fig. 2. In case 1, we treated with corticosteroid monotherapy (mPSL, $2 \mathrm{mg} / \mathrm{kg}$ daily) first for 2 weeks. We then added MMF (1,000 mg twice daily) because grade 3 HFT abnormalities persisted. HFT abnormalities improved to grade 2 or less by 2 weeks after the addition of MMF. Although she experienced abscess in the liver 1 month after the administration of immunosuppressive drugs, probably as a side effect of immunosuppression, the abscess was successfully treated and managed with antibiotics and percutaneous drainage.

Case 2 was treated with corticosteroid monotherapy (mPSL, $2 \mathrm{mg} / \mathrm{kg}$ daily), but HFT abnormalities did not improve. The patient died of acute exacerbation of chronic obstructive pulmonary disease (COPD) two months after the onset of nivolumab-related cholangitis.

Case 3 had grade 4 cholangitis as with case 1 , whose grade 4 cholangitis was refractory to initial systemic corticosteroid treatment. We worried about steroid refractory situation, and
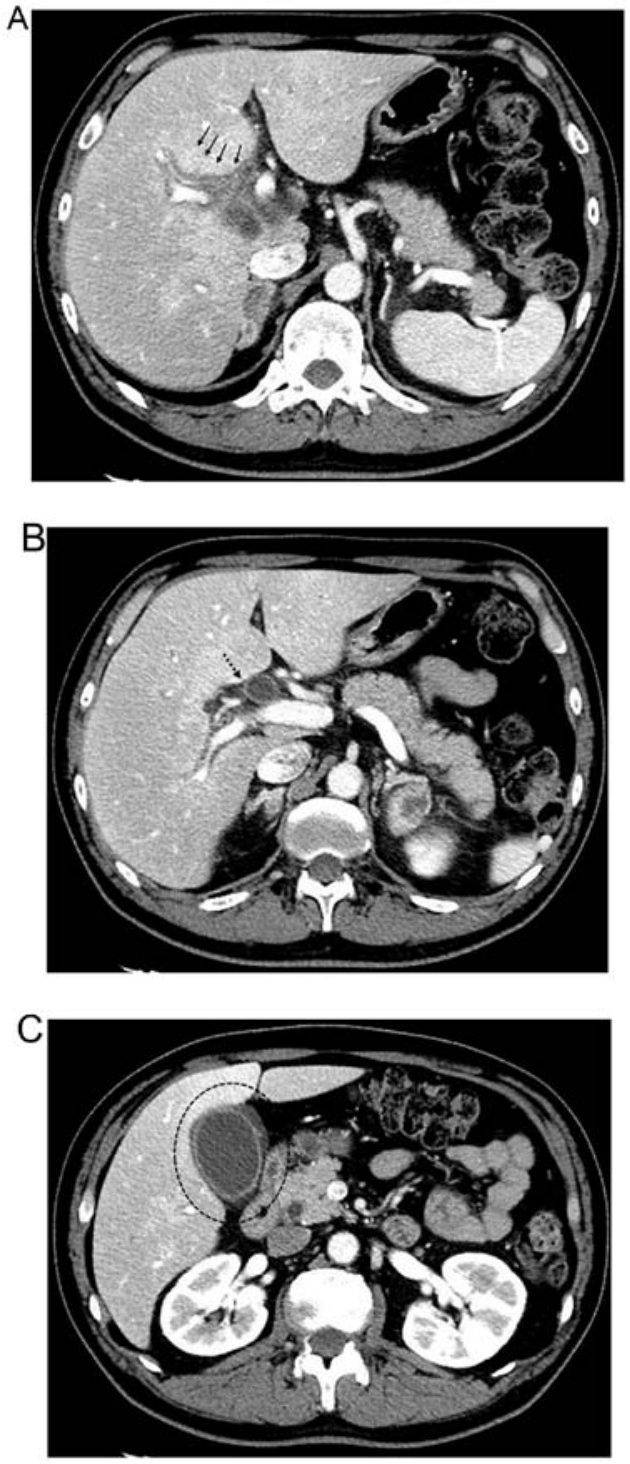

Figure 1. Contrast-enhanced computed tomography of a representative case at the onset of nivolumab-induced cholangitis. (A) Solid arrows indicate PE. (B) Dotted arrows indicate dilatation and hypertrophy of extrahepatic bile duct. (C) The circle indicates hypertrophy of gallbladder wall. PE, periportal edema. 
A
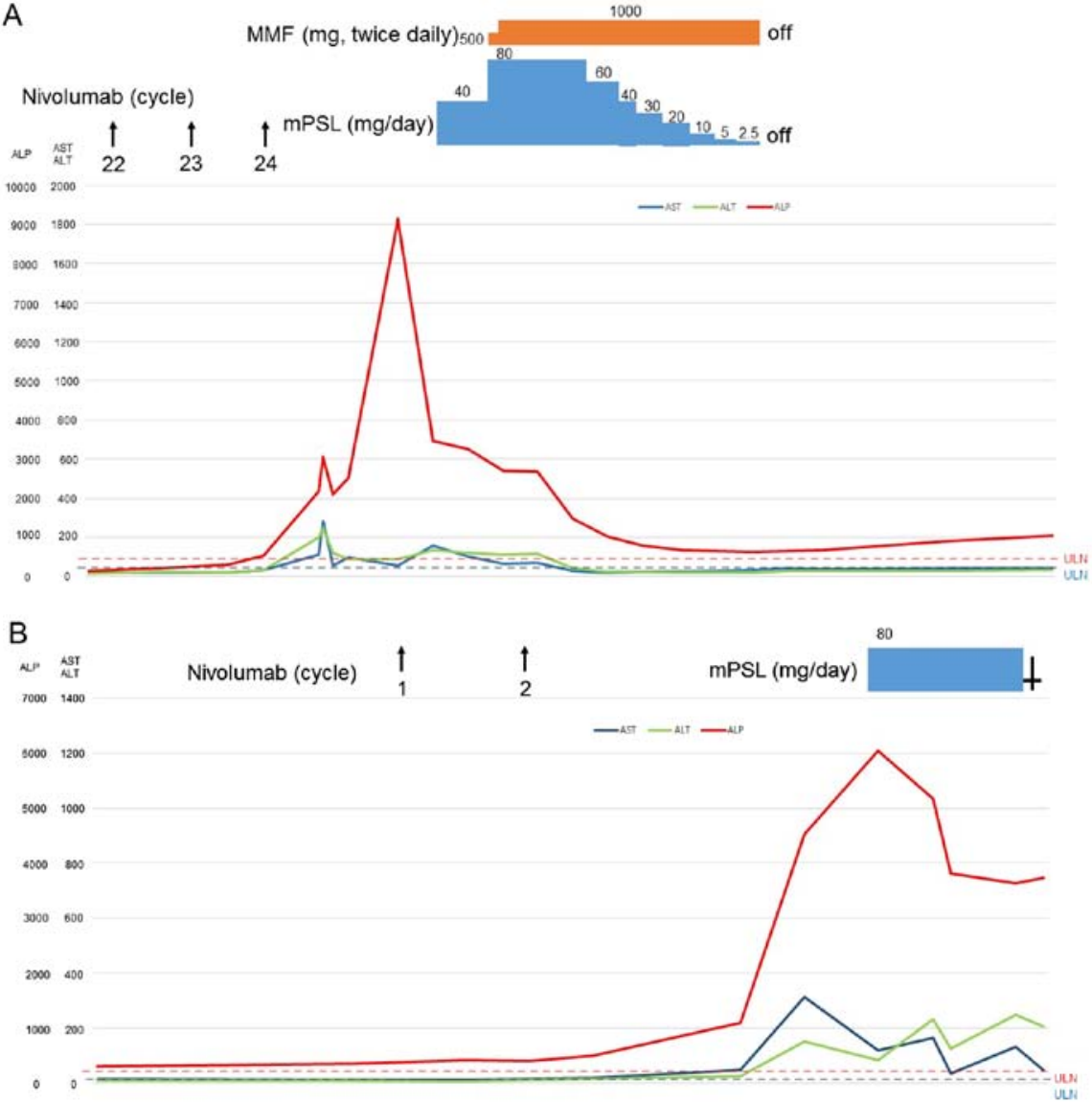

C
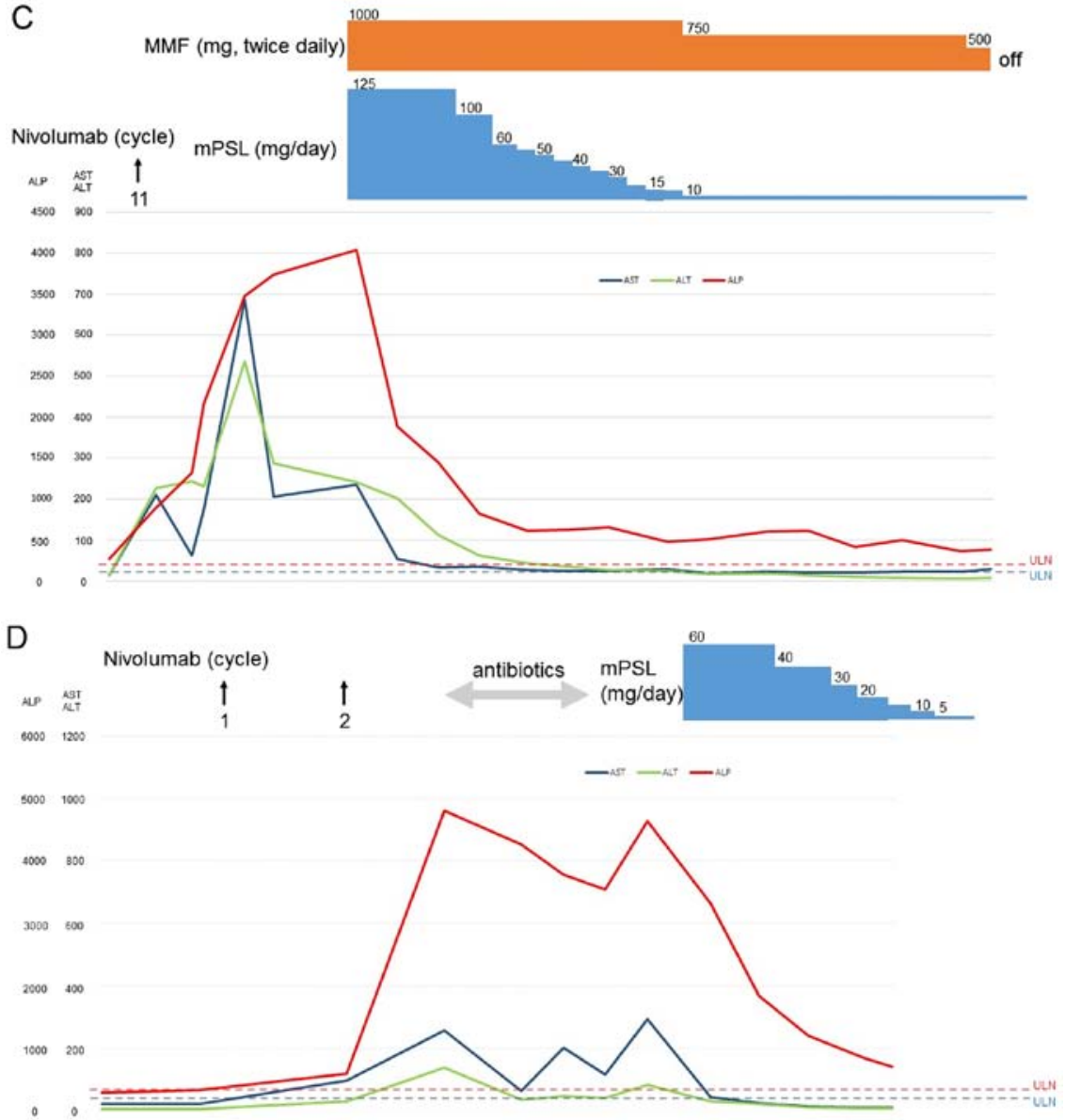

Figure 2. Hepatobiliary function test and clinical course of (A) case 1, (B) case 2, (C) case 3 and (D) case 4. ALP, alkaline phosphatase; ALT, alanine aminotransferase; AST, aspartate aminotransferase; MMF, mycophenolate mofetil; mPSL, methylprednisolone. 
Table III. Clinical manifestation and treatment of nivolumab-induced cholangitis.

\begin{tabular}{|c|c|c|c|c|c|c|c|c|}
\hline \multirow[b]{2}{*}{ Case } & \multirow[b]{2}{*}{ Symptoms } & \multirow[b]{2}{*}{ Grade } & \multicolumn{4}{|c|}{ Imaging results } & \multirow[b]{2}{*}{$\begin{array}{l}\text { Treatment } \\
\text { for cholangitis }\end{array}$} & \multirow[b]{2}{*}{$\begin{array}{l}\text { Improvement } \\
\text { of cholangitis }\end{array}$} \\
\hline & & & $\mathrm{PE}$ & $\begin{array}{l}\text { EHD } \\
\text { dilatation }\end{array}$ & $\begin{array}{l}\text { IHD } \\
\text { dilatation }\end{array}$ & $\begin{array}{l}\text { Hypertrophy } \\
\text { of EHD }\end{array}$ & & \\
\hline 1 & Low back pain & 4 & + & + & + & + & $\begin{array}{l}\mathrm{mPSL}(2.0 \mathrm{mg} / \mathrm{kg}) \\
\text { MMF }(500 \mathrm{mg}, \mathrm{bid}) \\
\text { EST, EBD+stent }\end{array}$ & Yes \\
\hline 2 & General fatigue, appetite loss & 3 & - & - & - & - & $\mathrm{mPSL}(2.0 \mathrm{mg} / \mathrm{kg})$ & No \\
\hline 3 & Epigastric pain & 4 & + & + & + & + & $\begin{array}{l}\mathrm{mPSL}(2.0 \mathrm{mg} / \mathrm{kg}) ; \\
\text { MMF ( } 500 \mathrm{mg}, \text { bid }) ; \mathrm{EST}\end{array}$ & Yes \\
\hline 4 & None & 3 & + & + & + & + & $\mathrm{mPSL}(2.0 \mathrm{mg} / \mathrm{kg})$ & Yes \\
\hline 5 & Fever, general fatigue & 3 & - & + & - & + & EBD+stent; Antibiotics & No \\
\hline 6 & Itching, jaundice & 4 & $\mathrm{~N} / \mathrm{A}$ & N/A & N/A & N/A & $\mathrm{mPSL}(1.0 \mathrm{mg} / \mathrm{kg})$ & Yes \\
\hline 7 & Fever, abdominal discomfort & 3 & - & + & - & + & PSL (0.5 mg/kg) & Yes \\
\hline 8 & $\begin{array}{l}\text { Fever, vomiting, abdominal } \\
\text { discomfort, diarrhea }\end{array}$ & 3 & - & + & - & + & $\begin{array}{l}\text { PSL }(0.5 \mathrm{mg} / \mathrm{kg}) \\
\text { EBD+stent }\end{array}$ & Yes \\
\hline 9 & Epigastric pain, soft stool & 3 & - & + & - & + & $\begin{array}{l}\text { EBD+stent; PSL } \\
(2.0 \mathrm{mg} / \mathrm{kg}) ; \text { Antibiotics }\end{array}$ & Yes \\
\hline 10 & Pruritic rash & $\mathrm{N} / \mathrm{A}$ & - & + & - & + & $\begin{array}{l}\text { PSL (60 mg/day) }->\text { mPSL } \\
(500 \mathrm{mg} / \text { day })\end{array}$ & No \\
\hline
\end{tabular}

PE, periportal edema; EHD, extrahepatic duct; IHD, intra-hepatic duct; mPSL, methyl-prednisolone; MMF, mycophenolate mofetil; PSL, prednisolone; EST, endoscopic sphinctectomy; EBD, endoscopic biliary drainage.

decided to treat case 3 with corticosteroid (mPSL, $1 \mathrm{mg} / \mathrm{kg}$ daily) and MMF (1,000 mg twice daily) simultaneously. After these drug administration, HFT abnormalities improved to grade 2 or less in 2 weeks without any immunosuppressive treatment-related complication.

In case 4 , bacterial infection in the neck was observed just after the onset of cholangitis; thus, this patient was first treated with antibiotics for cervical infection. Subsequently, she was treated with corticosteroid monotherapy (mPSL, $2 \mathrm{mg} / \mathrm{kg}$ daily) for nivolumab-induced cholangitis, and HFT abnormalities gradually improved.

Upon the improvement of HFT abnormalities, immunosuppressive agents were tapered gradually in three cases (all except case 2). Finally, the three cases whose cholangitis had improved were maintained with an equivalent dose of prednisolone $(\leq 10 \mathrm{mg})$, and MMF was successfully terminated. The recurrence of HFT abnormalities was not observed in any of the three cases. Moreover, none received subsequent chemotherapy or ICI treatment. Case 1 survived with SD at the data cut-off point without any subsequent treatment. Based on the literature review, we identified seven NSCLC patients with nivolumab-induced cholangitis (10-13).

\section{Discussion}

In this study, we present a rare case series of NSCLC with cholangitis induced by nivolumab, including two cases treated with a combination of corticosteroid and MMF. Immunotherapy with monoclonal antibodies targeting cytotoxic T lymphocyte-associated antigen 4 (CTLA4), as well as PD-1 and its ligand PD-L1, has become standard care in the treatment of NSCLC, with an increasing number of indications (6,7,14-16). Therefore, an increasing number of patients will be exposed to these drugs along with the risk of developing irAEs from these treatments. The incidence of irAE is high, with reports of rates of $32.0-64.8 \%$ in phase III clinical trials of nivolumab for advanced NSCLC. Among the irAEs, hepatotoxicity is observed in $1.5-7.7 \%$ of NSCLC patients treated with nivolumab, making it less common than skin-, gastrointestinal-, or endocrine-associated irAEs $(6,7)$. In particular, cholangitis induced by nivolumab is much less common, and there is little information available regarding the characteristics of patients who develop nivolumab-induced cholangitis or its clinical manifestations and treatment outcomes.

As summarized in Table II, including our four cases, we identified 10 NSCLC patients with cholangitis induced by nivolumab. The ages of these 10 patients ranged from 55 to 82 years (median age, 74 years), and the data set included six $(60.0 \%)$ men. Of the 10 patients, seven $(70.0 \%)$ had non-squamous NSCLC [adenocarcinoma: $n=6$, NSCLC-not otherwise specified (NOS): $n=1]$, and three (30.0\%) had squamous cell carcinoma. There were no apparent differences in the age distributions or the proportion of histological types between patients with nivolumab-induced cholangitis and other NSCLC cohorts (17).

The results of a recent phase III study of nivolumab in patients with non-squamous NSCLC showed that a greater RR was observed as PD-L1 expression increased (7), although in patients with squamous NSCLC, PD-L1 expression did not affect nivolumab efficacy (6). In addition, Haratani et al (18) reported that the development of irAEs is associated with the 
efficacy of nivolumab in patients with NSCLC. These results suggest the possibility of an association between the occurrence of irAEs, the response to nivolumab, and PD-L1 expression. In this study, the RR and DCR in patients with irAEs were higher than those in patients without irAEs, concomitant with positive PD-L1 expression. In addition, of the eight cases available for evaluation in terms of response to nivolumab treatment, four $(50.0 \%)$ achieved a PR and three (37.3\%) had $\mathrm{SD}$, reflecting higher rates than those achieved in previous clinical trials $(6,7)$.

The response to nivolumab in patients with cholangitis was comparable to that in patients with other irAEs, indicating the possibility that nivolumab-induced cholangitis occurs more frequently in patients with better responses to nivolumab, as with other irAEs. In other words, irAEs, including cholangitis, may occur particularly in patients who exhibit a better response to nivolumab treatment. However, these associations should be further investigated in future studies with larger sample sizes.

Although irAEs can occur at any time, most develop within a few weeks to a few months $(6,7,19)$. Skin-related irAEs can develop earlier, within 2-3 weeks after treatment, while hepatic irAEs develop within 6-7 weeks (19). In the 10 cases of nivolumab-induced cholangitis identified in this study, cholangitis occurred after 2-24 cycles (median, 5 cycles) of treatment, with three $(30.0 \%)$ occurring after more than 10 cycles of treatment. This indicates that ICI-induced cholangitis can also occur later, as with other hepatic irAEs. Clinicians should therefore keep in mind that a delayed effect of ICIs may occur during follow-up.

The dilatation and hypertrophy of the EHD were commonly observed according to imaging, being present in eight (88.8\%) of nine patients with imaging available for evaluation. PE was also identified commonly, being present in three (75\%) out of our four cases (Table III). Kim et al (20) reported that such findings could be observed in ICI-associated hepatitis caused by ipilimumab; however, both of these are non-specific and can be observed in other hepatic diseases. These results demonstrate the difficulty in distinguishing nivolumab-induced cholangitis from other hepatobiliary diseases using imaging results alone.

Cholangitis grade was severe in all nine patients with data available, exhibiting grade 3 in six $(66.6 \%)$ patients and grade 4 in three $(33.3 \%)$ patients (Table III). In general, the treatment for an irAE of grade 3 or above is the discontinuation of the suspected drugs and administration of systemic corticosteroids. When irAEs are steroid-refractory, other additional immunosuppressive agents should be considered (9). The optimal management of irAEs is based on clinical experience or in accordance with procedures for the management of autoimmune disease, as no prospective trials have been conducted to evaluate the best irAE treatment strategy. MMF is recommended for steroid-refractory hepatitis according to the ESMO guideline (9) and protocols of clinical trials for nivolumab for advanced NSCLC (6,7); however, there have been no case reports of NSCLC patients who experienced severe nivolumab-induced cholangitis and were treated with a combination of corticosteroid and MMF. To the best of our knowledge, our two cases with grade 4 cholangitis were the first NSCLC cases treated with a combination of corticosteroid and MMF for nivolumab-induced cholangitis. In these two cases, HFT abnormalities improved quickly after the administration of MMF combined with corticosteroid. Although the adverse effects of immunosuppressive treatment must be considered, especially that of infection, our cases suggest that this combination treatment is feasible and indeed effective against nivolumab-induced cholangitis. It remains to be clarified, however, whether simultaneous or sequential use of MMF is superior. However, owing to the rarity of this irAE, prospective trials to evaluate the best treatment strategy cannot be conducted. Therefore, further case reports are needed to establish the optimal management of patients with this irAE.

Limitations of this study include potential biases associated with the initiation, dosage, choice of immunosuppressive agents, and duration of immunosuppressive treatment, all of which are inevitable in a retrospective study. In addition, we were unable to perform histopathological analyses in the four cases from this study because of our retrospective design.

In conclusion, we have reported and reviewed a case series of NSCLC patients who developed cholangitis during ICI treatment. Nivolumab-induced cholangitis is a rare irAE that is more likely to develop in patients with better treatment responses to ICI. The combination of corticosteroid and MMF was an effective treatment in our two cases with grade 4 nivolumab-induced cholangitis, and its efficacy should be investigated in additional case reports.

\section{Acknowledgements}

Not applicable.

\section{Funding}

No funding was received.

\section{Availability of data and materials}

The datasets used and/or analyzed in the current study are not publicly available but are available from the corresponding author on reasonable request.

\section{Authors' contributions}

HI, TI and AYamas conceived and designed the study. HI, NT, MK, JK, KT, RO, KYaman, YT, AYamam, YS, MY, TS, KYamag, HM and AYamas acquired, analyzed and interpreted the data. HI drafted the manuscript. All authors read and approved the final manuscript.

\section{Ethics approval and consent to participate}

The study design was approved by the Institutional Review Board of Tottori University (approval no. 1054). Signed informed consents were obtained from the patients.

\section{Patient consent for publication}

Not applicable. 


\section{Competing interests}

The authors declare that they have no competing interests.

\section{References}

1. Siegel RL, Miller KD and Jemal A: Cancer statistics, 2019. CA Cancer J Clin 69: 7-34, 2019.

2. Pardoll DM: The blockade of immune checkpoints in cancer immunotherapy. Nat Rev Cancer 12: 252-264, 2012.

3. Chen Y,Mu CY and Huang JA: Clinical significance of programmed death-1 ligand-1 expression in patients with non-small cell lung cancer: A 5-year-follow-up study. Tumori 98: 751-755, 2012.

4. Velcheti V, Schalper KA, Carvajal DE, Anagnostou VK, Syrigos KN, Sznol M, Herbst RS, Gettinger SN, Chen L and Rimm DL: Programmed death ligand-1 expression in non-small cell lung cancer. Lab Invest 94: 107-116, 2014.

5. Topalian SL, Hodi FS, Brahmer JR, Gettinger SN, Smith DC, McDermott DF, Powderly JD, Carvajal RD, Sosman JA, Atkins MB, et al: Safety, activity, and immune correlates of anti-PD-1 antibody in cancer. N Engl J Med 366: 2443-2454, 2012.

6. Brahmer J, Reckamp KL, Baas P, Crinò L, Eberhardt WEE, Poddubskaya E, Antonia S, Pluzanski A, Vokes EE, HolgadoE, et al: Nivolumab versus docetaxel in advanced squamous-cell non-small-cell lung cancer. N Engl J Med 373: 123-135, 2015.

7. Borghaei H, Paz-Ares L, Horn L, Spigel DR, Steins M, Ready NE, Chow LQ, Vokes EE, Felip E, Holgado E, et al: Nivolumab versus docetaxel in advanced nonsquamous non-small-cell lung cancer. N Engl J Med 373: 1627-1639, 2015.

8. Michot JM, Bigenwald C, Champiat S, Collins M, Carbonnel F, Postel-Vinay S, Berdelou A, Varga A, Bahleda R, Hollebecque A, et al: Immune-related adverse events with immune checkpoint blockade: A comprehensive review. Eur J Cancer 54: 139-148, 2016.

9. Haanen JBAG, Carbonnel F, Robert C, Kerr KM, Peters S, Larkin $\mathrm{J}$ and Jordan K; ESMO Guidelines Committee: Management of toxicities from immunotherapy: ESMO Clinical Practice Guidelines for diagnosis, treatment and follow-up. Ann Oncol 28 (Suppl 4): iv119-iv142, 2017

10. Kawakami H, Tanizaki J, Tanaka K, Haratani K, Hayashi H, Takeda M, Kamata K, Takenaka M, Kimura M, Chikugo T, et al: Imaging and clinicopathological features of nivolumab-related cholangitis in patients with non-small cell lung cancer. Invest New Drugs 35: 529-536, 2017.

11. Gelsomino F, Vitale $G$ and Ardizzoni A: A case of nivolumab-related cholangitis and literature review: How to look for the right tools for a correct diagnosis of this rare immune-related adverse event. Invest New Drugs 36: 144-146, 2017.
12. Kashima J, Okuma Y, Shimizuguchi R and Chiba K: Bile duct obstruction in a patient treated with nivolumab as second-line chemotherapy for advanced non-small-cell lung cancer: A case report. Cancer Immunol Immunother 67: 61-65, 2018.

13. Kuraoka N, Hara K, Terai S, Yatabe Y and Horio Y: Peroral cholangioscopy of nivolumab-related (induced) ulcerative cholangitis in a patient with non-small cell lung cancer. Endoscopy 50: E259-E261, 2018.

14. Rittmeyer A, Barlesi F, Waterkamp D, Park K, Ciardiello F, von Pawel J, Gadgeel SM, Hida T, Kowalski DM, Dols MC, et al: Atezolizumab versus docetaxel in patients with previously treated non-small-cell lung cancer (OAK): A phase 3, open-label, multicentre randomised controlled trial. Lancet 389: 255-265, 2017.

15. Hellmann MD, Ciuleanu T-E, Pluzanski A, Lee JS, Otterson GA, Audigier-Valette C, Minenza E, Linardou H, Burgers S, Salman P, et al: Nivolumab plus ipilimumab in lung cancer with a high tumor mutational burden. N Engl J Med 378: 2093-2104, 2018.

16. Reck M, Rodríguez-Abreu D, Robinson AG, Hui R, Csôszi T, Fülöp A, Gottfried M, Peled N, Tafreshi A, Cuffe S, et al: Pembrolizumab versus chemotherapy for PD-L1-positive non-small-cell lung cancer. N Engl J Med 375: 1823-1833, 2016.

17. Abernethy AP, Arunachalam A, Burke T, McKay C, Cao X, Sorg R and Carbone DP: Real-world first-line treatment and overall survival in non-small cell lung cancer without known EGFR mutations or ALK rearrangements in US community oncology setting. PLoS One 12: e0178420, 2017.

18. Haratani K, Hayashi H, Chiba Y, Kudo K, Yonesaka K, Kato R, Kaneda H, Hasegawa Y, Tanaka K, Takeda M and Nakagawa K: Association of immune-related adverse events with nivolumab efficacy in non-small-cell lung cancer. JAMA Oncol 4: 374-378, 2017.

19. Weber JS, Kähler KC and Hauschild A: Management of immune-related adverse events and kinetics of response with ipilimumab. J Clin Oncol 30: 2691-2697, 2012.

20. Kim KW, Ramaiya NH, Krajewski KM, Jagannathan JP, Tirumani SH, Srivastava A and Ibrahim N: Ipilimumabassociated hepatitis: Imaging and clinicopathologic findings. Invest New Drugs 31: 1071-1077, 2013.

This work is licensed under a Creative Commons Attribution-NonCommercial-NoDerivatives 4.0 International (CC BY-NC-ND 4.0) License. 